\title{
Changes in Quality Characteristics of Different Deep Frying Fats During Frying and Regeneration Potentials of Different Adsorbents in Wasted Frying Oils
}

\author{
Yemiscioglu Fahri, Ozdikicierler Onur*, Onder Ozge \\ Food Engineering Department, Ege University, Izmir, Turkey \\ *Corresponding author: onur.ozdikicierler@ege.edu.tr
}

Received February 17, 2015; Revised February 27, 2015; Accepted March 16, 2015

\begin{abstract}
Regeneration potentials of synthetic magnesium silicate, acid activated bleaching earth and natural bleaching earth for the fried soybean oil after progressive laboratory scale frying. The wasted palm olein and sunflower oils which were obtained from two different restaurants were investigated. As indication for development of deterioration reactions in oil during frying and regeneration potentials of so-called adsorbents, free fatty acid content (FFA), total polar material (TPM), dielectric constant (DC) and color measurement values (L, a and b) were measured. According to the results, synthetic magnesium silicate (SMS), acid activated bleaching earth (ABE) and natural bleaching earth (NBE) have different adsorbent characteristics that were significantly affected by the origin, physical and chemical properties and production method of that adsorbent. Laboratory scale frying operations were significantly increased FFA, TPM and DC as a result of thermal oxidation reaction. The usage of SMS in the wasted soybean oil reduced FFA content, TPM and DC values while lighten the color. Nevertheless, the reductions of these values were much more evident for in wasted sunflower oil from Restaurant A (WA) and wasted palm olein from Restaurant B (WB) when compared to wasted oil obtained from laboratory scale frying operations. All values were reduced with the use of adsorbents while L values of WB is increased with adsorbent usage. Generally SMS showed a higher reducing potential on FFA content, TPM and DC values which are considered as deterioration indices of oil quality during frying operation.
\end{abstract}

Keywords: deep frying, adsorbent, regeneration of frying fats, total polar material, dielectric constant, free fatty acidity

Cite This Article: Yemiscioglu Fahri, Ozdikicierler Onur, and Onder Ozge, "Changes in Quality Characteristics of Different Deep Frying Fats During Frying and Regeneration Potentials of Different Adsorbents in Wasted Frying Oils." Journal of Food and Nutrition Research, vol. 3, no. 3 (2015): 176-181. doi: 10.12691/jfnr-3-3-8.

\section{Introduction}

Frying is one of the world's oldest and most common food preparation techniques and gives a flavor to the foods that are highly appreciated by consumers and is being extensively used in food processing [1,2,3].

During frying of foods, frying oil is exposed to air under high temperatures with the existence of water transported from food to frying oil. Major changes in food structure are oil uptake and water loss during frying while moisture content of frying oil increases. Presence of oxygen, the moisture content of the food, high oil temperature and the leaching of components from the food cause a variety of reactions to occur during frying including fat hydrolysis, fatty acid oxidation and dimer polymer formation all of which contribute to degradation of the frying oil $[1,4,5]$. Such degradation negatively affects the usability of frying oil negatively and oxidation products, such as peroxides, aldehydes, ketones, hydro peroxides, polymers and oxidized monomers can cause clinically harmful effects $[6,7,8,9]$. Once the oil is exposed to high frying temperatures, a portion of the triacylglycerol was converted to a myriad of degradation products. Total polar materials (TPM) are considered to be one of the most important substances for assessing degrading oils [6]. Increasing dielectric constant and acidity due to oxidation and hydrolysis reactions, are also expected degradation indices for frying oils. Color values (L, $a$ and $b$ ) of frying oil may change after consecutive frying operations depended on the conjugated food material $[1,10]$.

The frying oil may be reused several times before being discarded. In both industry and home, wasted frying oil disposal problems continue endanger environmental preservation in the point view of water and soil pollution aspects. Recycling used frying oils not only could reduce disposal problems, but also, more importantly, would increase the effective usage of food and agricultural resources [7].

The purification of the used frying oils with adsorbents may improve the quality of the oils and can be achieved by removing the undesirable oxidized, polar, color 
substances and polymers [6]. Synthetic magnesium silicate is an amorphous, insoluble white powder with a high specific surface area and is widely used as an analytical and industrial adsorbent and as an adsorbent to regenerate frying oils and purify biodiesel [12,13]. Many different adsorbent materials including activated carbon, clay, aluminum hydroxides, char-coal, celite, silica gel, silicon dioxide, and some other adsorbents have been, also, studied for this purpose [14].

The current study, regeneration potentials of synthetic magnesium silicate, acid activated bleaching earth and natural bleaching earth for used soybean frying oil obtained after progressive laboratory scale frying and palm olein and sunflower oils as wasted oils which were wasted oils obtained from two different restaurants were investigated. As indication for development of deterioration reactions in oil during frying and regeneration potentials of so-called adsorbents, FFA content, dielectric constant (DC), total polar material (TPM) content and color values were measured.

\section{Material and Methods}

\subsection{Material}

The source of refined soybean oil was used for progressive frying operationsin laboratory. Prior to starting frying operations in laboratory, FFA content, TPM, DC and color measurement parameters ("L”, “a” and "b" values) of soybean frying oil was $0.027 \%, 11.68 \%$, 1.325 and 2.51, $-0.19,0.22$ respectively. Frozen french fries were obtained from a local distributor in city Izmir, Turkey for frying operations.

Used sunflower oil (WA) was obtained from Restaurant A while used palm olein (WB) obtained from Restaurant $B$ where both restaurants are different local restaurants in city Izmir, Turkey. Wasted frying oils obtained from Restaurant A and B had been used for preparing of french fries. WB was used three days more than WA.

Synthetic Magnesium silicate (SMS), acid activated bleaching earth (ABE) (Bentonite - AMCOL Mineral AS, Istanbul, Turkey) and a natural bleaching earth (NBE) (GeohellasMak 200 Super Flo - Geohellas S.A., Athens, Greece) were used for regeneration trials.

\subsection{Methods}

Laboratory scale progressive frying operation was conducted with a 3L capacity household frier (Arcelik, Turkey). Total 60 frying times with $3 \mathrm{~kg}$ of soybean oil at $190 \pm 10^{\circ} \mathrm{C}$ were done. In every frying time $200 \mathrm{~g}$ of French fries was fried for 8 minutes. After every frying time, $50 \mathrm{ml}$ of frying oil was taken for determination of TPM content, FFA content, DC and color. Oil uptake was neglected and no fresh frying oil added after frying times. Remaining oil, after 60 frying times, was coded as wasted soybean frying oil (WSO) and regeneration trials were performed using this wasted oil.

Regeneration trials of WSO were performed by adding increasing amount of SMS (1\%, 3\%, 5\%, 7\% and 10\% of oil in weight) to WSO. WSO - adsorbent mixtures stirred at $100^{\circ} \mathrm{C}$ temperature using a magnetic stirrer for 15 minutes under atmospheric pressure. SMS was filtered from oils after treatment finished.
Regeneration trials of wasted frying oils (WA and WB) that were obtained from two different local restaurants, performed using SMS, ABE and NBE at different dosage levels $(0.3 \%, 0.6 \%, 1 \%, 2 \%$ and $3 \%$ of oil in weight) at $100^{\circ} \mathrm{C}$ temperature using a magnetic stirrer for 15 minutes under atmospheric pressure. Adsorbents were filtered from oils after treatment finished.

FFA content of oil samples was determined via IUPAC method 2.201. Results were represented as percentage of oleic acid [15].

DC value and TPM content of oil samples were determined using Food - Oil Sensor Oil Quality Analyzer probe (Testo 270 Cooking Oil Tester, USA). DC value and TPM content of oil samples were directly measured by dipping the probe to frying oil under constant temperature $\left(80^{\circ} \mathrm{C}\right)[16]$.

Hunter Lab was used to determine color values $(L, a, b)$ of oil samples using method AN 1005.00 [17].

Statistical evaluation of the resultswere carried out using IBM SPSS Statistics 20 for Windows.

\section{Results and Discussion}

\subsection{Laboratory Scale Fryings}

TPM and DC are the chemical indexes used to determine the degree of oxidative degradation of oil and is excellent predictors for food quality of many operations. FFA content and color values changes, also, during frying operation. FFA content, TPM content, DC and color measurement parameters ("L”, “a” and "b" values) of soybean oil can be seen in Table 1.

Table 1. hange of TPM content, DC, FFA content and color values (Hunter $L, a, b)$ in soybean oil along 60 frying operation times

\begin{tabular}{|c|c|c|c|c|c|c|}
\hline Frying Count & $\begin{array}{c}\text { FFA (oleic } \\
\text { acid, \%) }\end{array}$ & $\begin{array}{c}\text { TPM } \\
(\%)\end{array}$ & DC & L & $\mathrm{a}$ & $\mathrm{b}$ \\
\hline 0 & 0.03 & 11.68 & 1.33 & 2.51 & -0.20 & 0.22 \\
\hline 5 & 0.23 & 12.17 & 1.42 & 2.67 & -0.88 & 1.31 \\
\hline 10 & 0.28 & 13.26 & 1.44 & 4.33 & -1.84 & 1.82 \\
\hline 15 & 0.39 & 13.72 & 1.50 & 5.99 & -2.02 & 4.87 \\
\hline 20 & 0.38 & 13.88 & 1.84 & 8.61 & -1.37 & 7.41 \\
\hline 25 & 0.52 & 14.41 & 1.90 & 7.63 & -1.67 & 4.12 \\
\hline 30 & 0.60 & 15.33 & 1.97 & 4.07 & -1.13 & 2.41 \\
\hline 35 & 0.65 & 16.97 & 1.89 & 4.42 & -1.87 & 5.09 \\
\hline 40 & 0.70 & 17.57 & 2.08 & 5.63 & -1.44 & 5.74 \\
\hline 45 & 0.72 & 18.74 & 2.08 & 4.80 & -1.45 & 5.84 \\
\hline 50 & 0.93 & 19.58 & 2.04 & 4.85 & -1.58 & 5.94 \\
\hline 55 & 1.02 & 20.97 & 2.16 & 7.82 & 0.12 & 10.00 \\
\hline 60 & 1.01 & 21.97 & 2.73 & 9.28 & 2.27 & 12.84 \\
\hline
\end{tabular}

According to the results in Table 1, TPM and DC were increased with progressive frying times showing a linear tendency as expected. The correlation between values were analyzed using Pearson Correlation method (Table 2).

FFA content, TPM and DC; which are commonly used indices to detect wasted oil deterioration level; showed significant correlations with higher $\mathrm{r}$ values according to the Pearson correlation table given in Table 2. In this study, frying operations were carried out at $190 \pm 10^{\circ} \mathrm{C}$ which is the commonly preferred the frying temperature. The thermal impact on frying media initiated oxidation reaction and oxidated products started to form in oil. These oxidated products, which known as peroxides, hydroperoxides and light organic acids, show polar 
character and increase the TPM content and DC values. As polar materials increase in oil, the water solubility of oil should increase which unwillingly causing the hydrolysis of triglycerides and increasing FFA content. This chain effect continued through sequential frying operations reaching TPM content to 21.96, DC to 2.72 and FFA content to $1 \%$ after 60 frying. These findings are in accordance with studies carried out on relationships between frying time, FFA, DC and TPM in wasted palm and soybean frying oils $[18,19]$.

Table 2. Pearson correlation coefficients between various quality parameters of WSO

\begin{tabular}{|c|c|c|c|c|c|c|}
\hline & FFA & $L$ & $a$ & $b$ & TPM & DC \\
\hline \multirow{3}{*}{ FFA } & & $\mathrm{r}=0.553$ & $\mathrm{r}=0.373$ & $\mathrm{r}=0.816$ & $r=0.955$ & $r=0.910$ \\
\hline & & $\mathrm{p}<0.001$ & $\mathrm{p}=0.019$ & $\mathrm{p}<0.001$ & $\mathrm{p}<0.001$ & $\mathrm{p}<0.001$ \\
\hline & & $n=39$ & $\mathrm{n}=39$ & $\mathrm{n}=39$ & $n=39$ & $n=39$ \\
\hline \multirow{3}{*}{$\mathrm{L}$} & $\mathrm{r}=0.553$ & & $r=0.396$ & $r=0.846$ & $r=0.543$ & $r=0.659$ \\
\hline & $\mathrm{p}<0.001$ & & $\mathrm{p}=0.013$ & $\mathrm{p}<0.001$ & $\mathrm{p}<0.001$ & $\mathrm{p}<0.001$ \\
\hline & $n=39$ & & $n=39$ & $n=39$ & $n=39$ & $n=39$ \\
\hline \multirow{3}{*}{$\mathrm{a}$} & $r=0.373$ & $r=0.396$ & & $\mathrm{r}=0.576$ & $r=0.516$ & $\mathrm{r}=0.551$ \\
\hline & $\mathrm{p}=0.019$ & $p=0.013$ & & $\mathrm{p}<0.001$ & $p=0.001$ & $\mathrm{p}<0.001$ \\
\hline & $n=39$ & $n=39$ & & $\mathrm{n}=39$ & $n=39$ & $n=39$ \\
\hline \multirow{3}{*}{ b } & $\mathrm{r}=0.816$ & $\mathrm{r}=0.846$ & $r=0.576$ & & $\mathrm{r}=0.853$ & $\mathrm{r}=0.852$ \\
\hline & $\mathrm{p}<0.001$ & $\mathrm{p}<0.001$ & $\mathrm{p}<0.001$ & & $\mathrm{p}<0.001$ & $\mathrm{p}<0.001$ \\
\hline & $n=39$ & $n=39$ & $n=39$ & & $n=39$ & $n=39$ \\
\hline \multirow{3}{*}{ TPM } & $\mathrm{r}=0.955$ & $r=0.543$ & $r=0.516$ & $r=0.853$ & & $r=0.930$ \\
\hline & $\mathrm{p}<0.001$ & $\mathrm{p}<0.001$ & $\mathrm{p}=0.001$ & $\mathrm{p}<0.001$ & & $\mathrm{p}<0.001$ \\
\hline & $n=39$ & $\mathrm{n}=39$ & $\mathrm{n}=39$ & $n=39$ & & $n=39$ \\
\hline \multirow{3}{*}{ DC } & $r=0.910$ & $r=0.659$ & $\mathrm{r}=0.551$ & $r=0.852$ & $r=0.930$ & \\
\hline & $\mathrm{p}<0.001$ & $\mathrm{p}<0.001$ & $\mathrm{p}<0.001$ & $\mathrm{p}<0.001$ & $\mathrm{p}<0.001$ & \\
\hline & $n=39$ & $n=39$ & $n=39$ & $\mathrm{n}=39$ & $n=39$ & \\
\hline
\end{tabular}

a FFA (free fatty acid content); "L”, "a”, "b” (HunterLab color values); TPM (total polar matter); DC (dielectric constant)

${ }^{\mathrm{b}} \mathrm{r}$ is Pearson correlation coefficient significant at $\mathrm{p} \leq 0.05$ or $\mathrm{p} \leq 0.01$; $p$ is the probability; $n$ is number of samples

Color valuesof the tested sampleschangedduring frying operations. This changecan be observed through monitoring Pearson's correlation coefficients (Table 2). Change in "L", “a” and "b” values didn't show linear tendency although color of wasted oil was apperently darkened after frying operations.The Maillard reactions did not determine in the current study and the paragraph should be rewrite to be a confirmation of other study such as [12].

\subsection{Regeneration Trials of WSO using SMS}

Results of regeneration tests of WSO can be seen in Table 3 and Figure 1.

Table 3. Changesin TPM content and DC values of the regenerated sample by different dosage levels of SMS

\begin{tabular}{|c|c|c|}
\hline sample by different dosage levels of SMS \\
\hline Dosage & TPM $(\%)$ & DC \\
\hline Initial & $22^{\mathrm{a}} \pm 0$ & $2.74^{\mathrm{a}} \pm 0.07$ \\
\hline $1 \%$ & $20^{\mathrm{b}} \pm 0$ & $2.60^{\mathrm{a}, \mathrm{b}} \pm 0.06$ \\
\hline $3 \%$ & $18^{\mathrm{b}, \mathrm{c}} \pm 1$ & $2.50^{\mathrm{b}} \pm 0.07$ \\
\hline $5 \%$ & $18^{\mathrm{c}, \mathrm{d}} \pm 1$ & $2.22^{\mathrm{c}} \pm 0.06$ \\
\hline $7 \%$ & $17^{\mathrm{c}, \mathrm{d}} \pm 1$ & $2.00^{\mathrm{d}} \pm 0.10$ \\
\hline $10 \%$ & $17^{\mathrm{d}} \pm 0$ & $2.03^{\mathrm{d}} \pm 0.05$ \\
\hline
\end{tabular}

*Values were given with standard errors.

* Letters on columns show statistical difference according to Tukey's post-hoc test.
After SMS treatment of WSO, color values and FFA content has showed specific expected behaviors. Reduction of TPM and DC was found to be statistically important. Moreover a bleaching effect was achieved in appearance of wasted frying oil as a result of SMS application. 1\% of SMS was adequate to achieve such a reduction and possibly due to rapid saturation of adsorbent surface with coloring bodies. Free fatty acids were adsorbed as well as coloring bodies. Using $10 \%$ of adsorbent reduced $83 \%$ of FFA content $(1.03 \%$ to $0.17 \%$ in oleic acid) which is a very significant effect since many legislation set a limit on FFA content of wasted frying oil for determining replacement time of oil. Figure 1 shows the changes in FFA, TPM content, DC value and color values " $L$ ", “ $a$ ", “ $b$ " with respect to different SMS dosages. Numerious studies showed that active carbon can be used with magnesium silicate to enhance the FFA reducing effect and a 1:1 ratio of active carbon and magnesium silicate reduced nearly half of the FFA content [21].
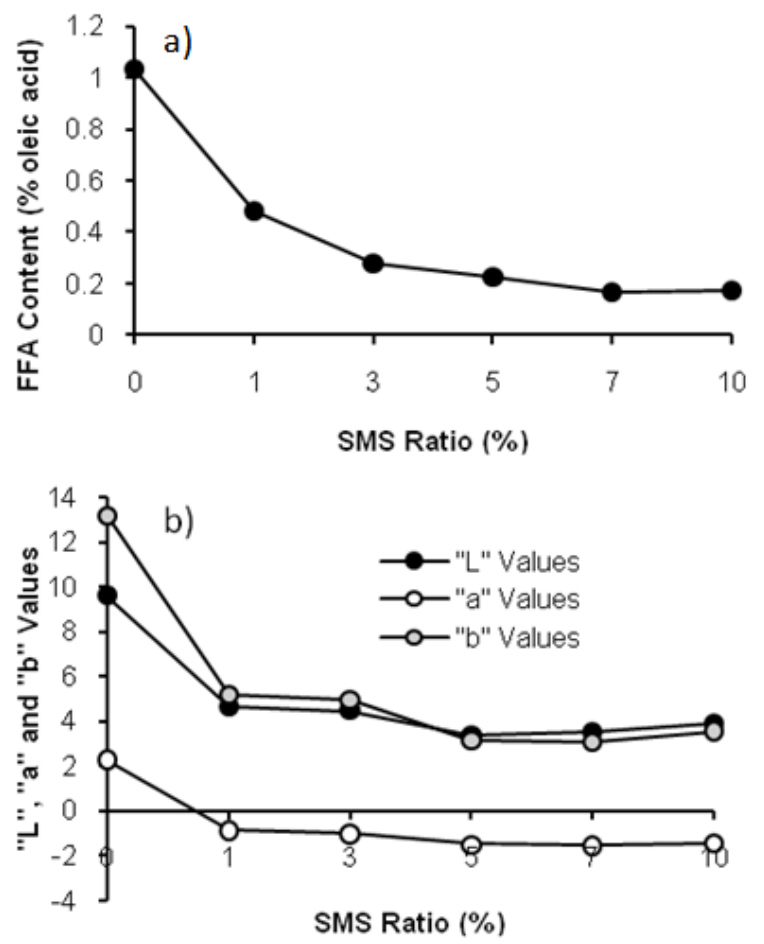

Figure 1. Change in FFA content (a), and color values ( $L$, $a$ and $b$ ) (b) with respect to SMS ratio in degenerated WSO

\subsection{Regeneration Trials of WA and WB Using Different Adsorbents}

The used palm olein and sunflower oil were collected from Restaurant A and Restaurant B respectively. Both restaurants are known as fast food restaurants that using frying oil to prepare potato fries. Regeneration trials carried out with different adsorbents. Synthetic Magnesium silicate (SMS), acid activated bleaching earth $(\mathrm{ABE})$ and natural bleaching earth (NBE) was compared according to reducing effects on FFA content, TPM content and DC as well as color values for wasted frying oils WA and WB. SMS is widely used for regeneration of wasted frying oils and commercially sold. ABE is used as a general bleaching earth having carotenoid adsorbance property and NBE having chlorophyll adsorbance property. 
The properties of wasted and the regenerated oils are presented in Table 4.

During deep fat frying, surface water of food were rapidlyvaporizes to form a crispy texture on the top layer. Hydrolysis reaction is known to be propagated by the water that had transferred from food matrix to frying oil during frying. Frying oil was exposed to water at prolonged usage periods therefore high FFA formation due to hydrolysis consequently. Consequently WB had been used three days more than WA. This situation explains the FFA difference ( $0.33 \%$ oleic acid at WA and $1.45 \%$ oleic acid at WB) between wasted frying oils. FFA content of both wasted oils was reduced but because of the FFA content was initially low ( $0.33 \%$ oleic acid) in WA, FFA reduction in WA wasn't distinctive as WB. TPM content and DC value was $20.45 \%$ and $2.72 \%$, respectively at WA while same values were $28.25 \%$ and $3.81 \%$, respectively at $\mathrm{WB}$ for initial condition.

Table 4. Change in the properties of wasted and the regenerated oilsby different adsorbents dosages

\begin{tabular}{|c|c|c|c|c|c|c|c|c|c|c|c|c|c|}
\hline & \multicolumn{7}{|c|}{ Restaurant A (Wasted Sunflower Oil) (WA) } & \multicolumn{6}{|c|}{ Restaurant B (Wasted Palm Olein) (WB) } \\
\hline & & FFA & TPM & DC & $L$ & $a$ & $\boldsymbol{b}$ & FFA & TPM & DC & $\boldsymbol{L}$ & $a$ & $\boldsymbol{b}$ \\
\hline & Initial & $0.33^{\mathrm{a}, \mathrm{b}}$ & $20.75^{\mathrm{a}, \mathrm{b}}$ & $2.72^{\mathrm{a}}$ & $18.35^{\mathrm{a}}$ & $1.29^{\mathrm{a}}$ & $8.4^{\mathrm{a}}$ & $1.45^{\mathrm{a}}$ & $28.25^{\mathrm{a}}$ & $3.81^{\mathrm{a}}$ & $7.78^{\mathrm{a}}$ & $0.18^{\mathrm{a}}$ & $2.93^{\mathrm{a}}$ \\
\hline \multirow{6}{*}{ SMS } & $0.3 \%$ & $\begin{array}{c}0.34^{\mathrm{a}} \\
\sigma=0.01\end{array}$ & $\begin{array}{c}19.79^{b} \\
\sigma=0.48\end{array}$ & $\begin{array}{c}2.54^{\mathrm{a}, \mathrm{b}} \\
\sigma=0.05\end{array}$ & $\begin{array}{c}11.99^{b} \\
\sigma=0.58\end{array}$ & $\begin{array}{c}-1.22^{\mathrm{b}} \\
\sigma=0.00\end{array}$ & $\begin{array}{c}1.52^{\mathrm{C}} \\
\sigma=0.06\end{array}$ & $\begin{array}{c}1.04^{\mathrm{b}} \\
\sigma=0.03\end{array}$ & $\begin{array}{c}22.12^{\mathrm{C}} \\
\sigma=0.20\end{array}$ & $\begin{array}{c}3.16^{\mathrm{b}} \\
\sigma=0.06\end{array}$ & $\begin{array}{c}9.66^{\mathrm{b}, \mathrm{c}} \\
\sigma=0.30\end{array}$ & $\begin{array}{c}-0.41^{\mathrm{c}} \\
\sigma=0.01\end{array}$ & $\begin{array}{c}2.19^{b} \\
\sigma=0.13\end{array}$ \\
\hline & $0.6 \%$ & $\begin{array}{c}0.33^{\mathrm{a}, \mathrm{b}, \mathrm{c}} \\
\sigma=0\end{array}$ & $\begin{array}{c}19.38^{\mathrm{b}} \\
\sigma=0.56\end{array}$ & $\begin{array}{c}2.55^{\mathrm{a}, \mathrm{b}} \\
\sigma=0.12\end{array}$ & $\begin{array}{c}6.82^{\mathrm{d}} \\
\sigma=0.15\end{array}$ & $\begin{array}{c}-1.52^{\mathrm{c}} \\
\sigma=0.05\end{array}$ & $\begin{array}{c}1.49^{\mathrm{c}} \\
\sigma=0.06\end{array}$ & $\begin{array}{c}1.00^{\mathrm{b}} \\
\sigma=0.03\end{array}$ & $\begin{array}{c}24.48^{\mathrm{b}} \\
\sigma=0.96\end{array}$ & $\begin{array}{c}3.01^{\mathrm{b}, \mathrm{c}} \\
\sigma=0.11\end{array}$ & $\begin{array}{c}9.32^{\mathrm{b}} \\
\sigma=0.06\end{array}$ & $\begin{array}{c}-0.32^{\mathrm{b}} \\
\sigma=0.01\end{array}$ & $\begin{array}{c}2.20^{\mathrm{b}} \\
\sigma=0.06\end{array}$ \\
\hline & $1 \%$ & $\begin{array}{c}0.31^{\mathrm{b}, \mathrm{c}} \\
\sigma=0.01\end{array}$ & $\begin{array}{c}22.24^{\mathrm{a}} \\
\sigma=1.39\end{array}$ & $\begin{array}{c}2.50^{\mathrm{b}} \\
\sigma=0.08\end{array}$ & $\begin{array}{c}5.86^{\mathrm{c}} \\
\sigma=0.12\end{array}$ & $\begin{array}{c}-1.74^{\mathrm{d}} \\
\sigma=0.07\end{array}$ & $\begin{array}{c}1.6^{\mathrm{c}} \\
\sigma=0.05\end{array}$ & $\begin{array}{c}1.03^{\mathrm{b}} \\
\sigma=0.05\end{array}$ & $\begin{array}{c}22.70^{\mathrm{b}, \mathrm{c}} \\
\sigma=1.02\end{array}$ & $\begin{array}{c}2.98^{\mathrm{b}, \mathrm{c}, \mathrm{d}} \\
\sigma=0.11\end{array}$ & $\begin{array}{c}9.72^{\mathrm{b}, \mathrm{c}} \\
\sigma=0.45\end{array}$ & $\begin{array}{c}-0.35^{\mathrm{b}} \\
\sigma=0.01\end{array}$ & $\begin{array}{c}2.21^{\mathrm{b}} \\
\sigma=0.05\end{array}$ \\
\hline & $2 \%$ & $\begin{array}{c}0.31^{\mathrm{c}} \\
\sigma=0.01\end{array}$ & $\begin{array}{c}16.20^{\mathrm{C}} \\
\sigma=0.67\end{array}$ & $\begin{array}{c}2.10^{\mathrm{c}} \\
\sigma=0.08\end{array}$ & $\begin{array}{c}7.58^{\mathrm{e}} \\
\sigma=0.12\end{array}$ & $\begin{array}{c}-1.34^{b} \\
\sigma=0.03\end{array}$ & $\begin{array}{c}2.29^{\mathrm{b}} \\
\sigma=0.08\end{array}$ & $\begin{array}{c}1.02^{\mathrm{b}} \\
\sigma=0.02\end{array}$ & $\begin{array}{c}20.93^{\mathrm{c}} \\
\sigma=0.74\end{array}$ & $\begin{array}{c}2.90^{\mathrm{c}, \mathrm{d}} \\
\sigma=0.01\end{array}$ & $\begin{array}{c}10.23^{\mathrm{c}} \\
\sigma=0.05\end{array}$ & $\begin{array}{c}-0.44^{\mathrm{c}} \\
\sigma=0.03\end{array}$ & $\begin{array}{c}2.02^{\mathrm{b,c}} \\
\sigma=0.10\end{array}$ \\
\hline & $3 \%$ & $\begin{array}{c}0.25^{\mathrm{d}} \\
\sigma=0.01 \\
\end{array}$ & $\begin{array}{c}17.06^{\mathrm{c}} \\
\sigma=0.27\end{array}$ & $\begin{array}{c}2.07^{\mathrm{C}} \\
\sigma=0.09\end{array}$ & $\begin{array}{c}5.23^{\mathrm{e}} \\
\sigma=0.02 \\
\end{array}$ & $\begin{array}{c}-1.97^{\mathrm{e}} \\
\sigma=0.07\end{array}$ & $\begin{array}{c}1.55^{\mathrm{c}} \\
\sigma=0.07 \\
\end{array}$ & $\begin{array}{c}0.97^{b} \\
\sigma=0.04\end{array}$ & $\begin{array}{c}20.94^{\mathrm{C}} \\
\sigma=0.61\end{array}$ & $\begin{array}{c}2.76^{\mathrm{d}} \\
\sigma=0.10\end{array}$ & $\begin{array}{c}10.05^{\mathrm{b}, \mathrm{c}} \\
\sigma=0.57\end{array}$ & $\begin{array}{c}-0.64^{\mathrm{d}} \\
\sigma=0.02\end{array}$ & $\begin{array}{c}1.92^{\mathrm{C}} \\
\sigma=0.07\end{array}$ \\
\hline & & FFA & TPM & DC & $L$ & $a$ & $b$ & FFA & TPM & DC & $L$ & $A$ & b \\
\hline \multirow{7}{*}{ ABE } & Initial & $0.33^{\mathrm{a}, \mathrm{b}}$ & $20.75^{\mathrm{a}, \mathrm{b}}$ & $2.72^{\mathrm{a}}$ & $18.35^{\mathrm{a}}$ & $1.29^{\mathrm{a}}$ & $8.4^{\mathrm{a}}$ & $1.45^{\mathrm{a}}$ & $28.25^{\mathrm{a}}$ & $3.81^{\mathrm{a}}$ & $7.78^{\mathrm{a}}$ & $0.18^{\mathrm{a}}$ & $2.93^{\mathrm{a}}$ \\
\hline & $0.3 \%$ & $\begin{array}{c}0.32^{b, c} \\
\sigma=0.01\end{array}$ & $\begin{array}{l}19.65^{\mathrm{a}, \mathrm{b}} \\
\sigma=0.13\end{array}$ & $\begin{array}{c}2.51^{\mathrm{b}} \\
\sigma=0.11\end{array}$ & $\begin{array}{c}9.79^{b} \\
\sigma=0.29\end{array}$ & $\begin{array}{c}-1.57^{\mathrm{e}} \\
\sigma=0.04\end{array}$ & $\begin{array}{c}2.86^{\mathrm{b}} \\
\sigma=0.03\end{array}$ & $\begin{array}{c}1.11^{\mathrm{b}} \\
\sigma=0.01\end{array}$ & $\begin{array}{c}24.22^{b} \\
\sigma=0.68\end{array}$ & $\begin{array}{c}2.83^{b} \\
\sigma=0.08\end{array}$ & $\begin{array}{c}9.44^{\mathrm{a}} \\
\sigma=0.19\end{array}$ & $\begin{array}{c}-0.15^{\mathrm{c}} \\
\sigma=0.01\end{array}$ & $\begin{array}{c}2.27^{\mathrm{c}, \mathrm{d}} \\
\sigma=0.04\end{array}$ \\
\hline & $0.6 \%$ & $\begin{array}{c}0.32^{\mathrm{a}, \mathrm{b}, \mathrm{c}} \\
\sigma=0.01\end{array}$ & $\begin{array}{l}19.45^{\mathrm{a}, \mathrm{b}} \\
\sigma=0.9\end{array}$ & $\begin{array}{c}2.21^{\mathrm{c}} \\
\sigma=0.06\end{array}$ & $\begin{array}{c}8.22^{\mathrm{c}} \\
\sigma=0.29\end{array}$ & $\begin{array}{c}-1.41^{\mathrm{d}} \\
\sigma=0.02\end{array}$ & $\begin{array}{c}1.44^{\mathrm{d}} \\
\sigma=0.02\end{array}$ & $\begin{array}{c}1.06^{\mathrm{b}} \\
\sigma=0.03\end{array}$ & $\begin{array}{c}25.42^{b} \\
\sigma=0.58\end{array}$ & $\begin{array}{c}2.53^{\mathrm{C}} \\
\sigma=0.09\end{array}$ & $\begin{array}{c}9.29^{\mathrm{a}} \\
\sigma=0.27\end{array}$ & $\begin{array}{c}-0.45^{\mathrm{f}} \\
\sigma=0.01\end{array}$ & $\begin{array}{c}2.11^{\mathrm{d}} \\
\sigma=0.1\end{array}$ \\
\hline & $1 \%$ & $\begin{array}{c}0.32^{\mathrm{a}, \mathrm{b}, \mathrm{c}} \\
\sigma=0.01\end{array}$ & $\begin{array}{c}16.38^{\mathrm{c}} \\
\sigma=0.94\end{array}$ & $\begin{array}{c}2.20^{\mathrm{c}} \\
\sigma=0.05\end{array}$ & $\begin{array}{c}7.55^{\mathrm{d}} \\
\sigma=0.18\end{array}$ & $\begin{array}{c}-1.29^{\mathrm{c}} \\
\sigma=0.05\end{array}$ & $\begin{array}{c}1.04^{\mathrm{e}} \\
\sigma=0.03\end{array}$ & $\begin{array}{c}1.52^{\mathrm{a}} \\
\sigma=0.04\end{array}$ & $\begin{array}{l}20.38^{\mathrm{d}} \\
\sigma=0.3\end{array}$ & $\begin{array}{c}2.55^{\mathrm{c}} \\
\sigma=0.08\end{array}$ & $\begin{array}{c}9.14^{\mathrm{a}} \\
\sigma=0.23\end{array}$ & $\begin{array}{c}-0.02^{\mathrm{b}} \\
\sigma=0\end{array}$ & $\begin{array}{c}2.38^{\mathrm{C}} \\
\sigma=0.11\end{array}$ \\
\hline & $2 \%$ & $\begin{array}{c}0.34 \mathrm{a} \\
\sigma=0.01\end{array}$ & $\begin{array}{c}18.13^{b} \\
\sigma=0.57\end{array}$ & $\begin{array}{c}2.16^{\mathrm{c}} \\
\sigma=0.12\end{array}$ & $\begin{array}{c}9.23^{\mathrm{b}} \\
\sigma=0.2\end{array}$ & $\begin{array}{c}-1.16^{\mathrm{b}} \\
\sigma=0.04\end{array}$ & $\begin{array}{c}2.65^{\mathrm{c}} \\
\sigma=0.16\end{array}$ & $\begin{array}{c}1.45^{\mathrm{a}} \\
\sigma=0.03\end{array}$ & $\begin{array}{c}22.06^{\mathrm{C}} \\
\sigma=0.72\end{array}$ & $\begin{array}{c}2.60^{\mathrm{c}} \\
\sigma=0.08\end{array}$ & $\begin{array}{c}8.23^{\mathrm{b}} \\
\sigma=0.41\end{array}$ & $\begin{array}{c}-0.35^{\mathrm{e}} \\
\sigma=0.01\end{array}$ & $\begin{array}{c}2.66^{\mathrm{b}} \\
\sigma=0.09\end{array}$ \\
\hline & $3 \%$ & $\begin{array}{c}0.31 c \\
\sigma=0.01\end{array}$ & $\begin{array}{c}18.47^{b} \\
\sigma=0.62\end{array}$ & $\begin{array}{c}2.10^{\mathrm{C}} \\
\sigma=0.02\end{array}$ & $\begin{array}{c}7.71^{\mathrm{c}, \mathrm{d}} \\
\sigma=0.27\end{array}$ & $\begin{array}{c}-1.25^{\mathrm{c}} \\
\sigma=0.02\end{array}$ & $\begin{array}{c}0.74^{\mathrm{f}} \\
\sigma=0.03\end{array}$ & $\begin{array}{c}1.43^{\mathrm{a}} \\
\sigma=0.08\end{array}$ & $\begin{array}{c}19.28^{\mathrm{d}} \\
\sigma=0.85\end{array}$ & $\begin{array}{c}2.57^{\mathrm{c}} \\
\sigma=0.09\end{array}$ & $\begin{array}{c}9.16^{\mathrm{a}} \\
\sigma=0.31\end{array}$ & $\begin{array}{c}-0.20^{\mathrm{d}} \\
\sigma=0\end{array}$ & $\begin{array}{c}2.45^{\mathrm{c}} \\
\sigma=0.02\end{array}$ \\
\hline & & FFA & TPM & DC & $L$ & $a$ & $b$ & FFA & TPM & DC & $L$ & $a$ & $b$ \\
\hline \multirow{6}{*}{ NBE } & Initial & $0.33^{\mathrm{a}}$ & $20.75^{a}$ & $2.72^{\mathrm{a}}$ & $18.35^{\mathrm{a}}$ & $1.29^{\mathrm{a}}$ & $8.40^{\mathrm{a}}$ & $1.45^{\mathrm{a}, \mathrm{b}, \mathrm{c}}$ & $28.25^{a}$ & $3.81^{\mathrm{a}}$ & $7.78^{\mathrm{a}}$ & $0.18^{\mathrm{a}}$ & 2.93a \\
\hline & $0.3 \%$ & $\begin{array}{c}0.34^{\mathrm{a}} \\
\sigma=0.01\end{array}$ & $\begin{array}{c}19.41^{\mathrm{a}, \mathrm{b}} \\
\sigma=0.82\end{array}$ & $\begin{array}{c}2.49^{\mathrm{a}, \mathrm{b}} \\
\sigma=0.16\end{array}$ & $\begin{array}{l}6.63^{\mathrm{b}, \mathrm{c}} \\
\sigma=0.1\end{array}$ & $\begin{array}{c}-1.61^{b} \\
\sigma=0.09\end{array}$ & $\begin{array}{c}1.94^{\mathrm{d}} \\
\sigma=0.01\end{array}$ & $\begin{array}{c}1.50^{\mathrm{a}} \\
\sigma=0.02\end{array}$ & $\begin{array}{c}22.10^{\mathrm{c}} \\
\sigma=0.93\end{array}$ & $\begin{array}{c}3.01^{\mathrm{b}} \\
\sigma=0.11\end{array}$ & $\begin{array}{c}9.41^{b} \\
\sigma=0.12\end{array}$ & $\begin{array}{l}-0.10^{\mathrm{C}} \\
\sigma=0\end{array}$ & $\begin{array}{c}2.37 b \\
\sigma=0.05\end{array}$ \\
\hline & $0.6 \%$ & $\begin{array}{c}0.33^{\mathrm{a}} \\
\sigma=0.01\end{array}$ & $\begin{array}{c}18.31^{b} \\
\sigma=0.69\end{array}$ & $\begin{array}{c}2.47^{b} \\
\sigma=0.04\end{array}$ & $\begin{array}{c}6.51^{\mathrm{b}, \mathrm{c}} \\
\sigma=0.25\end{array}$ & $\begin{array}{c}-1.59^{b} \\
\sigma=0.03\end{array}$ & $\begin{array}{c}2.54^{b} \\
\sigma=0.02\end{array}$ & $\begin{array}{c}1.36^{\mathrm{c}} \\
\sigma=0.05\end{array}$ & $\begin{array}{l}22.07^{\mathrm{C}} \\
\sigma=0.5\end{array}$ & $\begin{array}{c}2.97^{b} \\
\sigma=0.16\end{array}$ & $\begin{array}{c}9.36^{b} \\
\sigma=0.18\end{array}$ & $\begin{array}{l}-0.17^{\mathrm{e}} \\
\sigma=0\end{array}$ & $\begin{array}{c}2.20 b, c \\
\sigma=0.06\end{array}$ \\
\hline & $1 \%$ & $\begin{array}{l}0.30^{b} \\
\sigma=0\end{array}$ & $\begin{array}{l}18.84^{\mathrm{a}, \mathrm{b}} \\
\sigma=0.81\end{array}$ & $\begin{array}{c}2.38^{b} \\
\sigma=0.08\end{array}$ & $\begin{array}{c}5.96^{\mathrm{C}} \\
\sigma=0.3\end{array}$ & $\begin{array}{c}-1.76^{\mathrm{C}} \\
\sigma=0.03\end{array}$ & $\begin{array}{c}2.35^{\mathrm{c}} \\
\sigma=0.01\end{array}$ & $\begin{array}{c}1.47^{\mathrm{a}, \mathrm{b}} \\
\sigma=0.04\end{array}$ & $\begin{array}{c}19.04^{\mathrm{d}} \\
\sigma=0.48\end{array}$ & $\begin{array}{c}2.95^{\mathrm{b}} \\
\sigma=0.08\end{array}$ & $\begin{array}{c}9.18^{b} \\
\sigma=0.33\end{array}$ & $\begin{array}{c}-0.11^{\mathrm{d}} \\
\sigma=0.01\end{array}$ & $\begin{array}{c}2.32 b \\
\sigma=0.15\end{array}$ \\
\hline & $2 \%$ & $\begin{array}{c}0.30^{\mathrm{b}} \\
\sigma=0.01\end{array}$ & $\begin{array}{c}18.14^{\mathrm{b}} \\
\sigma=1.09\end{array}$ & $\begin{array}{c}2.33^{b} \\
\sigma=0.09\end{array}$ & $\begin{array}{c}5.97^{c} \\
\sigma=0.21\end{array}$ & $\begin{array}{c}-1.61^{b} \\
\sigma=0.02\end{array}$ & $\begin{array}{c}1.66^{\mathrm{e}} \\
\sigma=0.08\end{array}$ & $\begin{array}{c}1.40^{\mathrm{a}, \mathrm{b}, \mathrm{c}} \\
\sigma=0.04\end{array}$ & $\begin{array}{c}22.37^{\mathrm{c}} \\
\sigma=0.57\end{array}$ & $\begin{array}{c}2.88^{\mathrm{b}} \\
\sigma=0.07\end{array}$ & $\begin{array}{c}9.55^{\mathrm{b}} \\
\sigma=0.19\end{array}$ & $\begin{array}{c}-0.08^{\mathrm{b}} \\
\sigma=0\end{array}$ & $\begin{array}{c}2.28 \mathrm{~b} \\
\sigma=0.04\end{array}$ \\
\hline & $3 \%$ & $\begin{array}{c}0.30^{\mathrm{b}} \\
\sigma=0.01\end{array}$ & $\begin{array}{c}18.47^{\mathrm{b}} \\
\sigma=0.63\end{array}$ & $\begin{array}{c}2.30^{\mathrm{b}} \\
\sigma=0.07\end{array}$ & $\begin{array}{c}6.81^{\mathrm{b}} \\
\sigma=0.4\end{array}$ & $\begin{array}{c}-1.52^{\mathrm{b}} \\
\sigma=0.01\end{array}$ & $\begin{array}{c}1.85^{\mathrm{d}} \\
\sigma=0.06\end{array}$ & $\begin{array}{c}1.39^{\mathrm{b}, \mathrm{c}} \\
\sigma=0.02\end{array}$ & $\begin{array}{c}25.38^{b} \\
\sigma=1.2\end{array}$ & $\begin{array}{c}2.91^{\mathrm{b}} \\
\sigma=0.09\end{array}$ & $\begin{array}{c}10.38^{\mathrm{c}} \\
\sigma=0.37\end{array}$ & $\begin{array}{c}-0.21^{\mathrm{f}} \\
\sigma=0.01\end{array}$ & $\begin{array}{c}2.06 \mathrm{c} \\
\sigma=0.02\end{array}$ \\
\hline
\end{tabular}

* Letters on columns show the statistical difference according to Tukey test of reducing effect for each adsorbent separately.

Total polar material (TPM) is essentially non-TG fraction of the oil which is broadly grouped into polymerized and decomposed products based on molecular weight and polarity. The utility of dielectric property has been, also, increasingly recognized as a nondestructible, easy, quick, real time, and continuous method for evaluating changes in frying oils [10]. Regulations of European countries indicated $24-25 \%$ of TPM content is the replacement limit for frying and cooking oils $[7,8]$. In our study, statistical evaluation showed that all adsorbents caused similar reduction effects on TPM content for both wasted oils.

The value of $L$ indicates the level of luminosity or brightness, and found higher in WA (18.35 at WA and 7.78 at WB) at initial condition. Also "a” represents redness while "b” represent blueness. During frying, oil tents to be darkened as a result of heat effect which can be generally observed by measuring " $L$ " value. This darkening also increased “a” value.

\subsection{Regeneration Potential of SMS}

According to the results given in Table 3, the change in values wasn't apparent as WSO that was a model matrix. SMS, which was used for extending usage periods of frying oils in many countries, reduced FFA content of WA and WB significantly better than other two adsorbents, $\mathrm{ABE}$ and NBE. Since initial FFA content of WA was lower than WB, reduction of this value wasn't distinctive as WB. Moreover, according to the results in Figure 1 and Table 4, regardless from adsorbent amount, reduction of FFA content below $0.3 \%$ didn't observed in all wasted oils (WSO, WA, WB). This can be explained by the equilibrium concentration of FFA content for adsorption to SMS which is approximately $0.3 \%$ in our study. In a previous study, FFA reducing effect of SMS as a filter aid was studied and nearly $50 \%$ of reduction was achieved during filtration of model sunflower oil [22]. 
The current study showed that the SMS reduced TPM valuesfrom 20.75 to 17.06 for WA and from 28.25 to 20.94 for $\mathrm{WB}$ at $3 \%$ dosage level. On the other hand, a previous study indicated a better reducing effect of SMS for model sunflower oil which had been obtained by heating to $180^{\circ} \mathrm{C}$ for $4 \mathrm{~h}$ without food. This study indicated a decrease in TPM from $19 \%$ to $4 \%$, while TPM reducing effect of SMS didn't show any difference with diatomaceous earth, rice hull ash, wheat hull ash and barley hull ash, likewise results obtained in our study [22]. Also another study showed that SMS can reduce TPM content from $11 \%$ to $4.3 \%$ when the SMS amount was approximately $2 \%$ of wasted oil [13]. The synergetic adsorption effects of granular rhyolite and silicon dioxide with calcium silicate and magnesium silicate mixture was also investigated in another study. Although adsorbent granular rhyolite mixture reduced FFA content (from $0.69 \%$ to $0.15 \%$ ) more effective than the adsorbent mixture containing silicon dioxide, TPM reduction (from $9.9 \%$ to $3.9 \%$ ) was better with the silicon dioxide mixture[20]. These results showed that the mixture of different adsorbents may be used in frying oil regeneration operations to enhance adsorption capabilities.

SMS, also, showed significant effects on color values. Wherein, all adsorbents increased "L" values of WB and decreased "L" values of WA. "a" values were, also, significantly affected by SMS treatment. As SMS percentage increased, "a” values of WA and WB were decreased. This results show that redness of wasted oils were decreased during SMS treatment.

\subsection{Regeneration Potentials of ABE and NBE}

Usage of $\mathrm{ABE}$ and NBE didn't showed a significant effect on FFA content of both wasted frying oils. Utilization of $1 \%, 2 \%$ or $3 \%$ of NBE, resulted in a slight reduction in FFA content of WA and WB, while ABE caused an increase especially in case of WB. Because of the fact that $\mathrm{ABE}$ is acid activated, trace acid on adsorbent possibly reduced the FFA adsorbance capability. Also results in Table 4 showed that $\mathrm{ABE}$ has the highest reducing effect in DC of WA and WB according to Tukey's test. While NBE caused minor reductions in TPM content and DC as compared with the ABE.

After regeneration trials, NBE showed a high increasing effect on " $L$ " value in WA. Although equilibrium concentration of coloring bodies for adsorption on NBE is slightly higher than SMS, $63 \%$ of " $L$ " value (18.35 to 6.63) was reduced even at $0.3 \%$ of NBE content in WA. These results of " $L$ " values are in agreement with the results of Maskan et al. [5]. There was a slight reduction of "a" and "b" values in WB when using NBE as an adsorbent. In spite of SMS, increasing ABE and NBE percentages, it didn’t change “L” and “a” values.

\section{Conclusion}

Different adsorbents can be used for regeneration of frying oils. Different adsorbents have different adsorbent characteristics that were significantly affected by the origin, physical and chemical properties and production method of that adsorbent. In our study wasted frying oil regeneration capabilities of different adsorbents (SMS, $\mathrm{ABE}$ and NBE) was evaluated by means of change in FFA content, TPM, DC and color values. Results showed that, laboratory scale frying operations were increased FFA content, TPM and DC values as a result of thermal oxidation. The usage of SMS in this wasted soy oil (WSO) reduced FFA content, TPM and DC values while lighten the color and reducing the redness. Nevertheless, the reductions in wasted oils obtained from Restaurant A (WA) and Restaurant B (WB) were not as obvious as WSO. It can be followed from the results that among these adsorbents SMS showed a significant reduction capacity on FFA content and TPM value. SMS also recovered the changes of color values, especially in lightness and redness of oil, in WA and WB more effectively than ABE and NBE.

The visual recovery of a wasted frying oil cannot be only reason for reuse. Main problem of frying is that it provokes formation of polar matters and hydrolysis reaction in oil. Although SMS showed a good prospect for wasted oil recovery still reduction of TPM was not higher than $20 \%$ even it was used $3 \%$ of oil. There should be more studies in this field to investigate and enhance wasted oil recovery potentials of different adsorbents.

\section{References}

[1] Bulut E., Yilmaz E., "Comparison of the Frying Stability of Sunflower and Refined Olive Pomace Oils With/Without Adsorbent Treatment”, Journal of the American Oil Chemists Society, 87, 1145-1153, Oct.2010.

[2] Maskan M., Bagci H. I., "The recovery of used sunflower seed oil utilized in repeated deep-fat frying process”, European Food Research and Technology. 218, 26-31, Dec.2003.

[3] Wang F., Jiang L. Z., Zhu X. Q., Hou J. C., "Effects of frying on polar material and free fatty acids in soybean oils”, International Journal of Food Science and Technology. 48, 1218-1223, Jun.2013.

[4] Dobarganes C., Marquez-Ruiz G., Velasco J. "Interactions between fat and food during deep-frying”, European Journal of Lipid Science and Technology, 102, 521-528, Sep.2000.

[5] Maskan M., "Change in colour and rheological behaviour of sunflower seed oil during frying and after adsorbent treatment of used oil”, European Food Research and Technology. 218, 20-25, Dec.2003.

[6] Bhattacharya A. B., Sajilata M. G., Tiwari S. R., Singhal R. S. "Regeneration of thermally polymerized frying oils with adsorbents”, Food Chemistry., 110., 562-570., Oct.2008.

[7] Miyagi A., Nakajima M., "Regeneration of used frying oils using adsorption processing”, Journal of the American Oil Chemists Society. 80, 91-96, Jan.2003.

[8] Paul S., Mittal G. S., "Regulating the use of degraded oil/fat in deep-fat/oil food frying”, Critical Reviews in Food Science and Nutrition. 37, 635-662, 1997.

[9] Sun-Waterhouse D., Xue D. N., Wadhwa S., "Effects of Added Phenolics on the Lipid Deterioration and Antioxidant Content of Deep-Fried Potato Fritters”, Food and Bioprocess Technology. 6, 3256-3265, Nov.2013.

[10] Inoue C., Hagura Y., Ishikawa M., Suzuki K., "The dielectric property of soybean oil in deep-fat frying and the effect of frequency”, Journal of Food Science. 67, 1126-1129, Apr.2002.

[11] Buczek B., Chwialkowski W., "Purification of the used palm oil by adsorption”, Polish Journal of Chemical Technology. 10, 19-21, 2008.

[12] Faccini C.S., da Cunha M.E., Moraes M.S.A, Krause L.C., Manique M.C., Rodrigues M.R.A, Benvenutti E.V., Caramao E.B. "Dry Washing in Biodiesel Purification: a Comparative Study of Adsorbents” Journal of the Brazilian Chemical Society, 22, 558563, Jan.2011.

[13] Taspinar O. O., Ozgul-Yucel S., "Lipid adsorption capacities of magnesium silicate and activated carbon prepared from the same rice hull”, European Journal of Lipid Science and Technology. 110, 742-746, Aug.2008. 
[14] Dulger A., Yilmaz E. "Effectiveness of modified zeolites as adsorbent materials for frying oils" European Journal of Lipid Science and Technology, 115, 668-675, Jun.2013.

[15] IUPAC, Standard methods for the analysis of oils, fats and derivatives, International Union of Pure and Applied Chemistry, 1994.

[16] Testo, testo 270: Deep-frying Oil Tester instructional manual, Testo Limited, 2015.

[17] HunterLab "Hunterlab and CIE Lab Application Notes, HunterLab, 2012.

[18] Bou R., Navas J.A., Tres A., Codony R., Guardiola F., “Quality assessment of frying fats and fried snacks during continuous deepfat frying at different large-scale producers”, Food Control. 27, 254-267., Sep.2012.
[19] Freire P. C. M., Lobo L. C. B., Freitas G. D., Ferreira T. A. P. D., "Quality of deep frying oils and fats used in street-fairs in Goiania”, Brazil, Food Science and Technology. 33, 569-576, 2013.

[20] Lin S., Akoh C. C., Reynolds A. E., "Recovery of used frying oils with adsorbent combinations: refrying and frequent oil replenishment”, Food Research International. 34, 159-166, 2001.

[21] Buczek B., "Diesel Fuel from Used Frying Oil”, Scientific World Journal, 1-3., Jan.2014.

[22] Farag R. S., Basuny A. M. M., Arafat S. M., Arafa S. A. "Use of some agricultural waste hull ashes for the regeneration of fried sunflower oil”, International Journal of Food Science and Technology. 44, 1850-1856, Sep.2009. 